
\title{
R Reserach S Suare \\ Glycerol promoted the anaerobic production of rhamnolipids by different Pseudomonas aeruginosa strains
}

Feng Zhao ( $\sim$ zhaofeng2019@qfnu.edu.cn )

Qufu Normal University https://orcid.org/0000-0002-2808-6702

Chao Guo

Institute of Applied Ecology Chinese Academy of Sciences

Qingfeng Cui

Research Institute of Petroleum Exploration and Development

\section{Research}

Keywords: rhamnolipids, Pseudomonas aeruginosa, anaerobic biosynthesis, nitrate, crude glycerol, emulsifying activity

Posted Date: August 21st, 2020

DOl: https://doi.org/10.21203/rs.3.rs-60133/v1

License: (a) (1) This work is licensed under a Creative Commons Attribution 4.0 International License. Read Full License 


\section{Abstract}

Background: Rhamnolipids is the most widely studied and applied biosurfactants. The anaerobic biosynthesis of rhamnolipids has important research and practical significance, such as meeting the in situ production of biosurfactant in anoxic environments and the foamless fermentation of biosurfactants. A few studies have reported the anaerobic biosynthesis of rhamnolipids from rare Pseudomonas aeruginosa strains. What did promote the anaerobic biosynthesis of rhamnolipids, the specificity of the rare strains or the effect of specific substrates? Here, anaerobic production of rhamnolipids by different $P$. aeruginosa strains was investigated using diverse substrates. The anaerobic biosynthesis mechanism of rhamnolipids were also discussed from the substrate point of view.

Results: All $P$. aeruginosa strains anaerobically grew well using the tested substrates. But all $P$. aeruginosa strains anaerobically produced rhamnolipids only using substrates containing glycerol and nitrate. Fourier transform infrared (FTIR) spectra analysis confirmed the anaerobic production of rhamnolipids from all $P$. aeruginosa strains. All the anaerobically produced rhamnolipids decreased air-water surface tension from $72.6 \mathrm{mN} / \mathrm{m}$ to below $29.0 \mathrm{mN} / \mathrm{m}$ and emulsified crude oil with $\mathrm{El}_{24}$ above $65 \%$. Using crude glycerol as low-cost substrate, all $P$. aeruginosa strains can anaerobically grow and produce rhamnolipids to reduce the culture surface tension below $35 \mathrm{mN} / \mathrm{m}$. The glycerol metabolic intermediate, 1, 2-propylene glycol, can also achieve the anaerobic production of rhamnolipids by all $P$. aeruginosa strains.

Conclusions: Not the specificity of the rare $P$. aeruginosa strains but the effect of specific substrates promote the anaerobic biosynthesis of rhamnolipids by $P$. aeruginosa. Glycerol and nitrate are the excellent substrates for anaerobic production of rhamnolipids from all $P$. aeruginosa strains. Results indicated that glycerol metabolism involveed the anaerobic biosynthesis of rhamnolipids in $P$. aeruginosa. Results also showed the feasibility of using crude glycerol as low cost substrate to anaerobically biosynthesize rhamnolipids by $P$. aeruginosa.

\section{Background}

Rhamnolipids is the most widely studied and applied biosurfactants [1-3]. Due to its relatively high yield and good activity, rhamnolipids is promising in enhanced oil recovery, environmental bioremediation, agriculture, food, cosmetic, and other fields [3-7].

The anaerobic biosynthesis of rhamnolipids has important research and practical significance, such as meeting the in situ production of biosurfactant in anoxic environments and the foamless fermentation of biosurfactants [8]. Aerobic production of rhamnolipids fails to meet the in situ applications in the anoxic environments, such as deep soil, oil reservoirs, sediments [8-10]. The foam problem in biosurfactant fermentation has been perplexing the production of biosurfactants [11]. Foam is formed by biosurfactant enfolding air. Currently, the foam problem was solved by adding defoamer or collecting the produced foam, which also increased the risk of bacterial contamination $[11,12]$ Biosurfactants fermentation 
without aeration can avoid the foam problem. These breakthroughs require to further research the anaerobic synthesis of rhamnolipids, including the involved strain and culture medium.

Although the mainly rhamnolipids producers, Pseudomonas aeruginosa, are facultative bacteria $[8,13,14]$, only few studies have reported the anaerobic biosynthesis of rhamnolipids by rare $P$. aeruginosa strains $[9,10,15,16]$. P. aeruginosa can grow and metabolize at both aerobic and anaerobic conditions. But studies on production of rhamnolipids by $P$. aeruginosa were almost performed under aerobic conditions. Few studies reported that rare $P$. aeruginosa strains can anaerobically produce rhamnolipids $[8,17]$.

What did promote the anaerobic biosynthesis of rhamnolipids, the specificity of the rare strains or the effect of nutritional conditions? In previous studies, using facultative anaerobic and denitrifying bacterial strain $P$. stutzeri DQ1, the constructed engineering bacterial strain $P$. stutzeri Rhl achieved heterologous production of $P$. aeruginosa rhamnolipids under anaerobic conditions [18]. A wild-type $P$. aeruginosa SG that can anaerobically produce rhamnolipids was subsequently screened from production water of Xinjiang oil reservoir [9]. During the process of medium optimization, it was found that strain $P$. aeruginosa SG could anaerobically grew well using glucose and glycerol, but strain SG anaerobically produced rhamnolipids using glycerol not glucose [19]. Whether the anaerobic synthesis of rhamnolipids

is caused by the strain specificity of $P$. aeruginosa SG or the promoting effect of glycerol and nitrate? This study aims to analyze this problem by assessing the anaerobic production of rhamnolipids using different $P$. aeruginosa strains fed with diverse substrates. Results will deepen the biosynthesis theory of rhamnolipids and guide the process for anaerobic production of rhamnolipids.

In the present study, different $P$. aeruginosa strains were used. Diverse carbon sources and nitrogen sources were tested for anaerobic production of rhamnolipids by $P$. aeruginosa strains. Anaerobically produced rhamnolipids was confirmed by Fourier transform infrared (FTIR) spectra analysis. The physicochemical properties of rhamnolipids products were also evaluated. The feasibility of using crude glycerol as low cost substrate to anaerobically biosynthesize rhamnolipids by $P$. aeruginosa was studied. Prospects and bottlenecks existing in the anaerobic biosynthesis of rhamnolipids were also discussed. Results will guide to enhance anaerobic production of $P$. aeruginosa rhamnolipids by culture conditions development or strain improvement.

\section{Materials And Methods}

\section{Strains and culture conditions}

Four rhamnolipids-producing strains, $P$. aeruginosa strains (SG, L6-1, WJ1 and P4), were used in this study. Strains $P$. aeruginosa SG was used as positive control for anaerobic production of rhamnolipids [9]. Strain $P$. aeruginosa WJ1 was isolated from production fluid of Huabei oil reservoirs, China [20]. Strains $P$. aeruginosa SG, L6-1 and P4 were isolated from production fluid of Xinjiang oil reservoirs, China [9]. LB medium was used to prepare seed culture at $35^{\circ} \mathrm{C}$ and $180 \mathrm{rpm}$. For anaerobic production of rhamnolipids, four $P$. aeruginosa strains were cultured in $100 \mathrm{ml}$ serum bottles containing $80 \mathrm{ml}$ 
anaerobic medium with inoculum amount of $3 \%$, respectively. Briefly, the anaerobic medium was prepared as follows. The medium was boiled for $15 \mathrm{~min}$, and then $99.99 \%$ purity of $\mathrm{N}_{2}$ gas was injected into the boiling medium for $5 \mathrm{~min}$. Then medium was sub-packaged in serum bottles when it was hot. Press the rubber plug and press the aluminum cap under $\mathrm{N}_{2}$ gas protection. After sterilization and cooling to $30^{\circ} \mathrm{C}$, deoxidizer $\mathrm{Na}_{2} \mathrm{~S} \cdot 9 \mathrm{H}_{2} \mathrm{O}$ was added into the medium in serum bottles to a final concentration of $0.02 \%(\mathrm{w} / \mathrm{v})$ [18]. The anaerobic fermentation experiments were performed at $35^{\circ} \mathrm{C}$ and $50 \mathrm{rpm}$ for 10 days. Three parallel experiments were set for each bacterial strain. The non-inoculated medium was used as the negative control. The medium inoculated with strains $P$. aeruginosa SG was used as positive control.

\section{Analytical methods}

Culture samples were taken from serum bottles using sterile syringes. Bacterial strains biomass was represented by $\mathrm{OD}_{600}$ values of the culture samples. Culture samples were centrifuged at $10,000 \mathrm{~g}$ for 10 minutes. The surface tension of supernatant was measured by surface tensiometer (BZY-1, Shanghai Hengping Instrument and Meter Factory, Shanghai, China). The diameter of oil spreading circle formed by culture supernatant was determined as previous reported [21]. The crude oil (7-Middle area, Xinjiang oilfield, China) was used. Emulsification index $\left(\mathrm{El}_{24}\right)$ was measured to evaluate the emulsifying activity of produced rhamnolipids products as previous study described [18]. $\mathrm{El}_{24}$ values were calculated as the height of the emulsified layer $(\mathrm{mm})$ divided by the total height of the liquid column $(\mathrm{mm})$ and multiplied by 100 .

\section{$P$. aeruginosa strains fed with different carbon sources}

The common substrates, glucose, sucrose, molasses, soybean oil, palmitic acid, glycerol and starch, were tested as carbon sources for the anaerobic production of rhamnolipids by four $P$. aeruginosa strains. Strains $P$. aeruginosa SG was used as positive control for anaerobic production of rhamnolipids. The concentrations of carbon sources in the medium were all $40 \mathrm{~g} / \mathrm{l}$. The medium except carbon source contained $4 \mathrm{~g} / \mathrm{l}$ of $\mathrm{NaNO}_{3}, 4 \mathrm{~g} / \mathrm{l}$ of $\mathrm{K}_{2} \mathrm{HPO}_{4} \cdot 3 \mathrm{H}_{2} \mathrm{O}, 3 \mathrm{~g} / \mathrm{l}$ of $\mathrm{KH}_{2} \mathrm{PO}_{4}, 1.0 \mathrm{~g} / \mathrm{l}$ of $\mathrm{MgSO}_{4} \cdot 7 \mathrm{H}_{2} \mathrm{O}, 0.5 \mathrm{~g} / \mathrm{l}$ of KCl, $0.5 \mathrm{~g} / \mathrm{l}$ of $\mathrm{NaCl}, 0.2 \mathrm{~g} / \mathrm{l}$ of $\mathrm{CaCl}_{2} \cdot 2 \mathrm{H}_{2} \mathrm{O}$ and $5 \mathrm{ml}$ of trace elements solution. The trace elements solution contained $0.25 \mathrm{~g} / \mathrm{l}$ of $\mathrm{CuSO}_{4} \cdot 5 \mathrm{H}_{2} \mathrm{O}, 0.24 \mathrm{~g} / \mathrm{l}$ of $\mathrm{CaCl}_{2} \cdot 4 \mathrm{H}_{2} \mathrm{O}, 0.29 \mathrm{~g} / \mathrm{l}$ of $\mathrm{ZnSO}_{4} \cdot 7 \mathrm{H}_{2} \mathrm{O}, 0.17 \mathrm{~g} / \mathrm{l}$ of $\mathrm{MnSO}_{4} \cdot \mathrm{H}_{2} \mathrm{O}$. After the anaerobic fermentation process, the $\mathrm{OD}_{600}$, surface tension and oil spreading activity of culture samples were analyzed. Anaerobic production of rhamnolipids by strains was defined as that the surface tension of anaerobic culture was reduced to lower than $40 \mathrm{mN} / \mathrm{m}$ and the oil spreading circles diameters formed by anaerobic culture were greater than $10 \mathrm{~mm}$.

\section{$P$. aeruginosa strains fed with possible metabolic intermediates and analogues of glycerol}


The possible metabolic intermediates and analogues of glycerol (hydroxyacetone, 1, 2-propylene glycol and 1, 3-propylene glycol) were also evaluated for the anaerobic production of rhamnolipids by four $P$. aeruginosa strains. After the anaerobic fermentation process, the $\mathrm{OD}_{600}$, surface tension and oil spreading activity of culture samples were analyzed.

\section{$P$. aeruginosa strains fed with different nitrogen sources}

The common nitrogen nutrients, $\mathrm{NH}_{4} \mathrm{Cl}, \mathrm{NaNO}_{3}$, yeast extract and peptone, were tested as nitrogen sources for the anaerobic production of rhamnolipids by four $P$. aeruginosa strains. Strains $P$. aeruginosa SG was used as positive control for anaerobic production of rhamnolipids. The concentrations of nitrogen sources in the medium were all $4 \mathrm{~g} / \mathrm{L}$. The medium except nitrogen source contained $40 \mathrm{~g} / \mathrm{l}$ of glycerol, $4 \mathrm{~g} / \mathrm{l}$ of $\mathrm{K}_{2} \mathrm{HPO}_{4} \cdot 3 \mathrm{H}_{2} \mathrm{O}, 3 \mathrm{~g} / \mathrm{l}$ of $\mathrm{KH}_{2} \mathrm{PO}_{4}, 1.0 \mathrm{~g} / \mathrm{l}$ of $\mathrm{MgSO}_{4} \cdot 7 \mathrm{H}_{2} \mathrm{O}, 0.5 \mathrm{~g} / \mathrm{l}$ of KCl, $0.5 \mathrm{~g} / \mathrm{l}$ of NaCl, 0.2 $\mathrm{g} / \mathrm{l}$ of $\mathrm{CaCl}_{2} \cdot 2 \mathrm{H}_{2} \mathrm{O}$ and $5 \mathrm{ml}$ of trace elements solution. After the anaerobic fermentation process, the $\mathrm{OD}_{600}$, surface tension and oil spreading activity of culture samples were analyzed. Anaerobic production of rhamnolipids by strains was defined as that the surface tension of anaerobic culture was reduced to lower than $40 \mathrm{mN} / \mathrm{m}$ and the oil spreading circles diameters formed by anaerobic culture were greater than $10 \mathrm{~mm}$.

\section{$P$. aeruginosa strains fed with different the complex nitrogen sources}

The complex nitrogen sources of $\mathrm{NaNO}_{3}$ and yeast extract $(3: 7,5: 5,7: 3)$ were also tested for the anaerobic production of rhamnolipids by four $P$. aeruginosa strains. The amounts of complex nitrogen sources of $\mathrm{NaNO}_{3}$ and yeast extract was $4 \mathrm{~g} / \mathrm{l}$. After the anaerobic fermentation process, the $\mathrm{OD}_{600}$, surface tension and oil spreading activity of culture samples were analyzed.

\section{Rhamnolipids extraction from the anaerobic culture}

The anaerobiacally produced rhamnolipids were extracted as previous described [10]. Briefly, the cell free anaerobic culture broth was heated at $80^{\circ} \mathrm{C}$ for $30 \mathrm{~min}$ to denature the extracellular proteins. Samples were centrifuged at $10,000 \mathrm{~g}$ for 10 minutes. Using $2 \mathrm{~mol} / \mathrm{I}$ of $\mathrm{HCl}$-water solution, the $\mathrm{pH}$ value was adjusted to 2.0. Chloroform and methanol organic solvent (v/v, 2:1) was used for rhamnolipids extraction. The rhamnolipids products were extracted from the organic phase using a rotary evaporator $\left(50 \mathrm{rpm}, 45^{\circ} \mathrm{C}\right)$.

\section{FTIR spectra analysis}


Anaerobically produced rhamnolipids was confirmed by FTIR spectra analysis using rhamnolipids produced by strain $P$. aeruginosa SG as positive control. Briefly, $10 \mathrm{mg}$ of rhamnolipids extract and $90 \mathrm{mg}$ of $\mathrm{KBr}$ was mixed to make the translucent pellet with pressure of $25 \mathrm{Mpa}$ for $25 \mathrm{~s}$. A NICOLET $380 \mathrm{FTIR}$ spectrometer was used to record the FTIR spectra of the translucent pellet with the wave number from $400 \mathrm{~cm}^{-1}$ to $4000 \mathrm{~cm}^{-1}[10,18]$.

\section{$P$. aeruginosa strains fed with crude glycerol and nitrate}

To further reduce the medium cost, crude glycerol instead of glycerol was assessed for the anaerobic production of rhamnolipids by four $P$. aeruginosa strains. The concentration of crude glycerol in the medium was $40 \mathrm{~g} / \mathrm{L}$. The medium except crude glycerol contained $4 \mathrm{~g} / \mathrm{l}$ of $\mathrm{NaNO}_{3}, 4 \mathrm{~g} / \mathrm{l}$ of $\mathrm{K}_{2} \mathrm{HPO}_{4} \cdot 3 \mathrm{H}_{2} \mathrm{O}$, $3 \mathrm{~g} / \mathrm{l}$ of $\mathrm{KH}_{2} \mathrm{PO}_{4}, 1.0 \mathrm{~g} / \mathrm{l}$ of $\mathrm{MgSO}_{4} \cdot 7 \mathrm{H}_{2} \mathrm{O}, 0.5 \mathrm{~g} / \mathrm{l}$ of KCl, $0.5 \mathrm{~g} / \mathrm{l}$ of $\mathrm{NaCl}, 0.2 \mathrm{~g} / \mathrm{l}$ of $\mathrm{CaCl}_{2} \cdot 2 \mathrm{H}_{2} \mathrm{O}$ and $5 \mathrm{ml}$ of trace elements solution. After the anaerobic fermentation process, the $\mathrm{OD}_{600}$, surface tension and oil spreading activity were analyzed.

\section{Results And Discussion}

\section{Anaerobic production of rhamnolipids by $P$. aeruginosa strains using different carbon sources}

Using the tested substrates as carbon sources, the results of biomass $\left(\mathrm{OD}_{600}\right)$, surface activity and oil spreading activity were shown in Fig. 1. At anaerobic conditions, four $P$. aeruginosa strains obtained the biomass with $\mathrm{OD}_{600}$ values between 1.80 to 4.00 using the tested substrates (Fig. 1a). Results indicated that four $P$. aeruginosa strains can grow well under anaerobic conditions using all the tested substrates. Results also confirmed that $P$. aeruginosa are facultative bacteria $[8,13,14]$. From the point of view of biomass, molasses, glucose and glycerol were more favorable to the anaerobic growth and reproduction of $P$. aeruginosa strains. Molasses, glucose and glycerol as water-soluble substrates may be more conducive to the rapid absorption and anaerobic metabolize by bacterial cells of $P$. aeruginosa.

As shown in Fig. 1b, the surface tension values of anaerobic culture were reduced to lower than $40 \mathrm{mN} / \mathrm{m}$ using soybean oil (from $45 \mathrm{mN} / \mathrm{m}$ to about $37 \mathrm{mN} / \mathrm{m}$ ), palmitic acid (from $49 \mathrm{mN} / \mathrm{m}$ to about $37 \mathrm{mN} / \mathrm{m}$ ) and glycerol (from $63 \mathrm{mN} / \mathrm{m}$ to about $32 \mathrm{mN} / \mathrm{m}$ ). Using glycerol, four $P$. aeruginosa strains reduced the surface tension of anaerobic culture with the greatest decrease $(49.5 \%)$ in the surface tension and the lowest surface tension values. As shown in Fig. 1c, the oil spreading circles diameters formed by anaerobic culture of four strains were greater than $10 \mathrm{~mm}$ using soybean oil (about $11 \mathrm{~mm}$ ), palmitic acid (about $11 \mathrm{~mm}$ ) and glycerol (about $20 \mathrm{~mm}$ ). The oil spreading circles diameters formed by bacterial culture can be used to indirectly characterize the biosurfactants yield of bacterial strain [21]. Results demonstrated that four $P$. aeruginosa strains can efficiently produce biosurfactants under anaerobic conditions using glycerol as carbon sources. 
Though all the tested substrates can be used as carbon sources for production of rhamnolipids by $P$. aeruginosa under aerobic conditions [22-25]. In this study, only glycerol can be used for anaerobic production of rhamnolipids by $P$. aeruginosa strains among the tested substrates. As previous studies described, strain $P$. aeruginosa SG anaerobically produced rhamnolipids using glycerol $[9,19]$. In this study, $P$. aeruginosa strains except strain SG also anaerobically produced rhamnolipids using glycerol as carbon source. Here, anaerobic production of rhamnolipids is not induced by the specificity of the rare $P$. aeruginosa (strain SG) but the nutrient factor of glycerol.

\section{Anaerobic production of rhamnolipids by $P$. aeruginosa strains using different nitrogen sources}

Using $\mathrm{NH}_{4} \mathrm{Cl}, \mathrm{NaNO}_{3}$, yeast extract and peptone as nitrogen sources, anaerobic cell growth and rhamnolipids production by $P$. aeruginosa strains were comparatively studied. The results of biomass $\left(\mathrm{OD}_{600}\right)$, surface activity and oil spreading activity were shown in Fig. 2. Anaerobic culture of four $P$. aeruginosa strains obtained the biomass with $\mathrm{OD}_{600}$ values between 2.50 to 4.50 using the inorganic nitrogen sources, organic nitrogen sources and compound nitrogen sources (Fig. 2a). Four $P$. aeruginosa strains can grow well under anaerobic conditions using different nitrogen sources. Results also confirmed that $P$. aeruginosa are facultative bacteria $[13,14]$. As shown in Fig. 2a, the organic nitrogen sources were more favorable to the anaerobic growth and reproduction of $P$. aeruginosa strains. The small molecular peptides contained in yeast extract and peptone may provide abundant and available nutrition for cell growth of $P$. aeruginosa [26].

As shown in Fig. 2b, the surface tension values of anaerobic culture were all reduced from $63 \mathrm{mN} / \mathrm{m}$ to lower than $40 \mathrm{mN} / \mathrm{m}$ using all tested nitrogen sources. Using $\mathrm{NaNO}_{3}$ as nitrogen sources, four $P$.

aeruginosa strains reduced the surface tension of anaerobic culture with the greatest decrease $(51.3 \%)$ in the surface tension and the lowest surface tension values of about $31 \mathrm{mN} / \mathrm{m}$. As shown in Fig. 2c, the oil spreading circles diameters formed by anaerobic culture of four strains were greater than $10 \mathrm{~mm}$ using all tested nitrogen sources. Using $\mathrm{NaNO}_{3}$ as nitrogen sources, anaerobic culture of four $P$. aeruginosa strains formed oil spreading circles with diameters about $20 \mathrm{~mm}$. Although four $P$. aeruginosa strains also obtained higher biomass using the complex nitrogen sources of $\mathrm{NaNO}_{3}$ and yeast extract (Fig. 2a), the anaerobic production of rhamnolipids were relatively less (Fig. 2c). Results demonstrated that $\mathrm{NaNO}_{3}$ was more favorable to the anaerobic production of rhamnolipids by $P$. aeruginosa. Studies also reported that organic nitrogen sources were not conducive to the synthesis of rhamnolipids [27]. Nitrate was considered to be the best nitrogen source for rhamnolipid production under aerobic conditions [28.29]. At anaerobic conditions, nitrate was not only the excellent nitrogen source for rhamnolipids production but also the good electron acceptor for anaerobic metabolism of $P$. aeruginosa. $P$. aeruginosa, as facultative bacteria, can use other electron acceptors except oxygen, such as nitrate [8,15], so $P$. aeruginosa can grow and metabolize at both aerobic and anaerobic conditions $[13,14]$. 


\section{FTIR spectra analysis of anaerobically produced rhamnolipids using glycerol and nitrate}

The anaerobically produced rhamnolipids from four $P$. aeruginosa strains using glycerol and nitrate were confirmed by FT-IR spectra, respectively. Rhamnolipids produced by strain $P$. aeruginosa SG was used as the positive control. As shown in Fig. 3, the FTIR spectra of the anaerobically produced rhamnolipids from $P$. aeruginosa strains WJ1, L6-1 and P4 were similar to that of rhamnolipids produced from strain $P$. aeruginosa SG. The FTIR spectra of the anaerobically produced rhamnolipids from four $P$. aeruginosa strains were also similar to the spectra of reported rhamnolipids $[30,31]$. All the FTIR spectra had the characteristic absorption bands around $2927 \mathrm{~cm}^{-1}, 2858 \mathrm{~cm}^{-1}$ and $1465 \mathrm{~cm}^{-1}$ causing by the $\mathrm{C}-\mathrm{H}$ stretching vibrations of aliphatic groups and the absorption bands around $1735 \mathrm{~cm}^{-1}$ causing by the ester groups. All the FTIR spectra also had the absorption area between $1452 \mathrm{~cm}^{-1}$ and $1045 \mathrm{~cm}^{-1}$ causing by the $\mathrm{C}-\mathrm{H}$ and $\mathrm{O}-\mathrm{H}$ vibrations that are typical vibrations for carbohydrates. Results showed that four $P$. aeruginosa strains did produce rhamnolipids under anaerobic conditions using glycerol and nitrate.

\section{Activity of anaerobically produced rhamnolipids using glycerol and nitrate}

The rhamnolipids-water solutions $(200 \mathrm{mg} / \mathrm{L})$ were prepared using the extracted rhamnolipids products. The air-water surface tension and the emulsification index $\left(\mathrm{El}_{24}\right)$ to crude oil was measured to evaluate their surface activity and emulsifying activity. All the anaerobically produced rhamnolipids using glycerol and nitrate decreased the air-water surface tension from $72.6 \mathrm{mN} / \mathrm{m}$ to lower than $29 \mathrm{mN} / \mathrm{m}$. Studies reported the aerobically produced rhamnolipids can decrease the air-water surface tension to lower than $27 \mathrm{mN} / \mathrm{m}$ [32]. In this study, the anaerobically produced rhamnolipids from $P$. aeruginosa exhibited excellent surface activity as well. Good surface activity helps to change contact angle, increase wetting activity and even facilitate wetting reversal. The excellent surface activity can also improve the capillary effect and facilitate the flow of groundwater or oil in porous media. These are of great significance for enhanced oil recovery and environmental pollution remediation [33]. Anaerobically produced rhamnolipids all showed better emulsifying activity to crude oil with $\mathrm{El}_{24}$ values higher than $65 \%$. Studies reported the aerobically produced rhamnolipids can emulsified crude oil with $\mathrm{El}_{24}$ values ranging from $53 \%$ to $90 \%[32,34]$. In this study, the anaerobically produced rhamnolipids from $P$. aeruginosa also exhibited good emulsifying activity, which would be significant to enhanced oil recovery and pollution remediation $[5,35,36]$. The good emulsifying activity of biosurfactants can assist oil dispersion in oil reservoir to reduce oil viscosity and oil flow $[37,38]$. Besides, the excellent emulsification effect can increase the solubility of hydrophobic pollutants in the environment and increase the bioavailability of hydrophobic pollutants [35,39]. 


\section{Anaerobic production of rhamnolipids by $P$. aeruginosa strains using crude glycerol}

Using crude glycerol as low-cost substrate, all $P$. aeruginosa strains can grow under anaerobic conditions and obtained biomass with $\mathrm{OD}_{600}$ values between 2.10 to 2.50. As shown in Fig. 4, four $P$. aeruginosa strains anaerobically produced rhamnolipids to reduce the culture surface tension below $35 \mathrm{mN} / \mathrm{m}$. Compared to using glycerol as carbon source, the diameters of oil spreading circles formed by four $P$. aeruginosa strains anaerobic culture were smaller using crude glycerol. Results showed that using crude glycerol for anaerobic production of rhamnolipids by $P$. aeruginosa is feasible. Previous studies reported aerobic production of rhamnolipids by $P$. aeruginosa using crude glycerol $[40,41]$. Using glycerol or crude glycerol, the rhamnolipids yields from $P$. aeruginosa were both lower under anaerobic conditions.

Enhancing the anaerobic production of rhamnolipids from $P$. aeruginosa is in demand. In future, it is a good choice to develop high-yielding strains of rhamnolipids through biosynthesis pathways regulation and functional genes manipulation. Identifying the biosynthesis pathways and key genes involved in anaerobic production of rhamnoipids are of great importance

\section{Research perspectives to the anaerobic biosynthesis mechanism of rhamnolipids}

Glycerol is water soluble, easy to be absorbed and used by microorganisms, and is an excellent metabolic substrate for microorganisms. In this study, only glycerol promoted the anaerobic production of rhamnolipids by $P$. aeruginosa. But the mechanism of anaerobic biosynthesis of rhamnolipids by $P$. aeruginosa using glycerol is still unclear. In this study, from the point of substrate, the possible metabolic intermediates and analogues of glycerol (hydroxyacetone, 1, 2-propylene glycol and 1, 3-propylene glycol) were also evaluated for the anaerobic production of rhamnolipids by $P$. aeruginosa strains. The results of $\mathrm{OD}_{600}$, surface activity and oil spreading activity were shown in Fig. 5. P. aeruginosa strains obtained the biomass with $\mathrm{OD}_{600}$ values between 2.00 to 3.00 using the possible metabolic intermediates and analogues and analogues of glycerol (Fig. 5a). Four $P$. aeruginosa strains can anaerobically grow well using the possible metabolic intermediates and analogues of glycerol. These substrates are water-soluble and easily enter into the glycolysis and gluconeogenesis pathways, which may be conducive to the rapid absorption and anaerobic metabolize by $P$. aeruginosa [42]. Among the possible metabolic intermediates and analogues of glycerol, only 1, 2-propylene glycol promoted four $P$. aeruginosa strains to produce rhamnolipids under anaerobic conditions, reducing the surface tension of anaerobic culture from 63.2 $\mathrm{mN} / \mathrm{m}$ to about $31 \mathrm{mN} / \mathrm{m}$ (Fig. 5b). Using 1, 2-propylene glycol, the oil spreading circles diameters formed by anaerobic culture of four strains were about $20 \mathrm{~mm}$ (Fig. 5c). Results demonstrated that 1, 2-propylene glycol, similar to glycerol, can promote $P$. aeruginosa to anaerobically biosynthesize rhamnolipids.

Here, results again confirmed that anaerobic production of rhamnolipids is not induced by the specificity of the rare $P$. aeruginosa (strain SG) but the specific substrates. Glycerol and 1, 2-propylene glycol can 
promote $P$. aeruginosa to anaerobically biosynthesize rhamnolipids. What is the mechanism of anaerobic rhamnolipids biosynthesis by $P$. aeruginosa metabolizing glycerol and 1, 2-propylene glycol? Hauser and

Karnovsky cultured $P$. aeruginosa strain using ${ }^{14} \mathrm{C}$-labeled glycerol- $-{ }^{14} \mathrm{C}$ and glycerol- $\beta-{ }^{14} \mathrm{C}$ under aerobic conditions. And they found that the $\mathrm{C}_{6}$ unit of rhamnose group in rhamnolipids product was directly condensed from the two molecules glycerol $\left(C_{3}\right.$ unit) without carbon chain rearrangement [44]. The polyol can be oxidized into dihydroxyacetone (DHA), then DHA can be converted into dihydroxyacetone phosphate (DHAP) which enters glycolysis and gluconeogenesis pathways $[42,43]$. This may be why $P$. aeruginosa can metabolize glycerol and 1, 2-propylene glycol for biosynthesis the precursors of rhamnolipids, rhamnose and fatty acids. But the biosynthesis pathways and key genes involved in anaerobic biosynthesis of rhamnoipids using glycerol is still unclear. Elucidating the mechanism of anaerobic biosynthesis of rhamnolipids is helpful to the development and application of strains high producing rhamnolipids.

The anaerobic biosynthesis of rhamnolipids has important research and practical significance. Anaerobic production of rhamnolipids can meet the in situ production of biosurfactant in anoxic environments, such as deep soil, oil reservoirs, sediments [8-10]. Based on anaerobic production of rhamnolipids, fermentation without aeration can avoid the foam problem in production of rhamnolipids. However, enhancing the anaerobic production yield of rhamnolipids is critical. Future research will be concentrate on elucidating the mechanism of anaerobic biosynthesis of rhamnolipids through revealing the pathways and key genes involved using transcriptome technology.

\section{Conclusions}

Tested $P$. aeruginosa strains efficiently produced rhamnolipids under anaerobic conditions. Not the specificity of the rare $P$. aeruginosa strains but the effect of specific substrates promote the anaerobic biosynthesis of rhamnolipids by $P$. aeruginosa. Glycerol and nitrate are the excellent substrates for anaerobic production of rhamnolipids from all $P$. aeruginosa strains. Glycerol metabolism promoted the anaerobic biosynthesis of rhamnolipids in $P$. aeruginosa. Crude glycerol can be used as low-cost substrate for anaerobically producing rhamnolipids by $P$. aeruginosa. Transcriptomic analysis would facilitate to reveal the metabolic pathways and key genes involved in anaerobic production of rhamnolipids in future.

\section{Declarations}

\section{Authors' contributions}

FZ conceived and designed the study, carried out the experiments and drafted the manuscript. CG participated in analyzing the data. QFC assisted in revising the manuscript. All authors read and approved the final manuscript. 


\section{Acknowledgements}

This work was financially supported by the National Natural Science Foundation of China (31700117).

\section{Competing interests}

The authors declare that they have no competing interests.

\section{Availability of data and materials}

The datasets supporting the conclusions of this article are included within the article.

\section{Consent for publication}

All the co-authors approved the publication of this work in Microbial Cell Factories.

\section{Funding}

This work was financially supported by the National Natural Science Foundation of China (31700117).

\section{References}

1. Müller MM, Kügler JH, Henkel M, Gerlitzki M, Hörmann B, Pöhnlein M, Syldatk C, Hausmann R. Rhamnolipids-next generation surfactants? J Biotechnol. 2012; 162:366-380.

2. Varjani S, Upasani VN. Critical review on biosurfactant analysis, purification and characterization using rhamnolipid as a model biosurfactant. Bioresour Technol, 2017; 232: 389-397.

3. Chong H, Li Q. Microbial production of rhamnolipids: opportunities, challenges and strategies. Microb Cell Fact. 2017; 16(1): 137.

4. Brown LR. Microbial enhanced oil recovery (MEOR). Curr Opin Microbiol.2010; 13:316-320.

5. Gudiña EJ, Rodrigues Al, Alves E, Domingues MR, Teixeira JA, Rodrigues LR. Bioconversion of agroindustrial by-products in rhamnolipids toward applications in enhanced oil recovery and bioremediation. Bioresour Technol. 2015; 177:87-93.

6. Cameotra SS, Singh P. Synthesis of rhamnolipid biosurfactant and mode of hexadecane uptake by Pseudomonas Microb Cell Fact. 2009; 8(1): 16.

7. Zou H, Du W, Ji M, Zhu R. Enhanced Electrokinetic Remediation of Pyrene-Contaminated Soil Through pH Control and Rhamnolipids Addition. Environ Eng Sci. 2016; 33(7):507-513.

8. Domingues PM, Almeida A, Leal LS, et al. Bacterial production of biosurfactants under microaerobic and anaerobic conditions. Reviews in Environmental Science and Biotechnology, 2017; 16(2): 239- 
272.

9. Zhao F, Zhang J, Shi R, Han S, Ma F, Zhang Y. Production of biosurfactant by a Pseudomonas aeruginosa isolate and its applicability to in situ microbial enhanced oil recovery under anoxic conditions. RSC Adv. 2015; 5(45):36044-36050.

10. Zhao F, Shi R, Ma F, et al. Oxygen effects on rhamnolipids production by Pseudomonas aeruginosa. Microb Cell Fact. 2018; 17(1): 39.

11. Anic I, Apolonia I, Franco P, et al. Production of rhamnolipids by integrated foam adsorption in a bioreactor system. 2018, AMB Expr, 8: 122.

12. Long $X$, Shen $C$, He N, et al. Enhanced rhamnolipids production via efficient foam-control using stop valve as a foam breaker. Bioresour Technol, 2017; 224: 536-543.

13. Arai H. Regulation and function of versatile aerobic and anoxic respiratory metabolism in Pseudomonas aeruginos Front Microbiol. 2011; 2:1-13.

14. Schobert M, Jahn D. Anaerobic physiology of Pseudomonas aeruginosa in the cystic fibrosis lung. Int J Med Microbiol. 2010; 300:549-556.

15. Chayabutra C, Wu J, Ju LK. Rhamnolipids Production by Pseudomonas aeruginosa Under Denitrification: Effects of Limiting Nutrients and Carbon Substrates. Biotechnol Bioeng. 2001; 72:2533.

16. Albino JD, Namb, IM. Partial characterization of biosurfactant produced under anaerobic conditions by Pseudomonas sp ANBIOSURF-1. Adv Mater Res. 2010; 93:623-626.

17. Shekhar S, Sundaramanickam A, Balasubramanian T. Biosurfactant producing microbes and their potential applications: A review. Crit Rev Environ Sci Technol. 2015; 45(14):1522-1554.

18. Zhao F, Shi R, Zhao J, et al. Heterologous production of Pseudomonas aeruginosa rhamnolipid under anaerobic conditions for microbial enhanced oil recovery. J Appl Microbiol, 2015; 118(2): 379-389.

19. Zhao F, Zhou J, Han S, Ma F, Zhang Y, Zhang J. Medium factors on anaerobic production of rhamnolipids by Pseudomonas aeruginosa SG and a simplifying medium for in situ microbial enhanced oil recovery applications. World J Microbiol Biotechnol. 2016; 32(4):54.

20. Xia WJ, Luo ZB, Dong HP, Yu L, Cui QF, Bi YQ. Synthesis, Characterization, and Oil Recovery Application of Biosurfactant Produced by Indigenous Pseudomonas aeruginosa WJ1 Using Waste Vegetable Oils. Appl Biochem Biotechnol. 2012; 166:1148-1166.

21. Zhao F, Liang X, Ban Y, Han S, Zhang J, Zhang Y, Ma F. Comparison of Methods to Quantify Rhamnolipids and Optimization of Oil Spreading Method. Tenside Surfact Det. 2016; 53(3):243-248.

22. Marsudi S, Unno H, Hori K, et al. Palm oil utilization for the simultaneous production of polyhydroxyalkanoates and rhamnolipids by Pseudomonas aeruginosa. Appl Microbiol Biotechnol. 2008, 78(6): 955-961.

23. Tan YN, Li Q. Microbial production of rhamnolipids using sugars as carbon sources. Microbial Cell Factories, 2018, 17(1): 1-13. 
24. Nicolò MS, Cambria MG, Impallomeni G, Rizzo MG, Pellicorio C, Ballistreri A, Guglielmino SP. Carbon source effects on the mono/dirhamnolipid ratio produced by Pseudomonas aeruginosa L05, a new human respiratory isolate. New Biotechnol. 2017; 39: 36-41.

25. Ehinmitola EO, Aransiola E F, Adeagbo OP, et al. Comparative study of various carbon sources on rhamnolipid production. South African Journal of Chemical Engineering, 2018, 26: 42-48.

26. Kerr ED, Schulz BL. Vegemite Beer: yeast extract spreads as nutrient supplements to promote fermentation. PeerJ, 2016, 4: e2271.

27. Reis, R.S., Pereira, A.G., Neves, B.C., Freire, D.M.G., 2011. Gene regulation of rhamnolipid production in Pseudomonas aeruginosa - A review. Technol. 102, 6377-6384.

28. Soberón-Chávez G, Lepine F, Déziel E. Production of rhamnolipids by Pseudomonas aeruginosa. Appl Microbiol Biotechnol. 2005; 68:718-725.

29. Reis RS, Rocha SLG, Chapeaurouge DA, Domont GB, Santa Anna LMM, Freire DMG, Perales J. Effects of carbon and nitrogen sources on the proteome of Pseudomonas aeruginosa PA1 during rhamnolipid production. Process Biochem 2010; 45: 1504-1510.

30. Leitermann F, Syldatk C, Hausmann R. Fast quantitative determination of microbial rhamnolipids from cultivation broths by ATR-FTIR Spectroscopy. J Biol Eng. 2008; 2:13-20.

31. Kiefer J, Radzuan M N, Winterburn J, et al. Infrared Spectroscopy for Studying Structure and Aging Effects in Rhamnolipid Biosurfactants. Applied Sciences, 2017; 7(5):533.

32. Nitschke M, Cost SGVAO, Contiero J. Rhamnolipid surfactants: an update on the general aspects of these remarkable biomolecules. Biotechnol Prog. 2005; 21(6):1593-1600.

33. Park T, Jeon M, Yoon S, et al. Modification of Interfacial Tension and Wettability in Oil-Brine-Quartz System by in Situ Bacterial Biosurfactant Production at Reservoir Conditions: Implications for Microbial Enhanced Oil Recovery. Energy \& Fuels, 2019; 33(6): 4909-4920.

34. Pekdemir T, Copur M, Urum K. Emulsification of crude oil-water systems using biosurfactants. Process saf environ. 2005; 83(1):38-46.

35. Rahman KSM, Rahman TJ, Kourkoutas Y, Petsas I, Marchant R, Banat IM. Enhanced bioremediation of $n$-alkane in petroleum sludge using bacterial consortium amended with rhamnolipids and micronutrients. Bioresour Technol. 2003; 90(2):159-168.

36. Varjani SJ, Upasani VN. Core Flood study for enhanced oil recovery through ex-situ bioaugmentation with thermo-and halo-tolerant rhamnolipids produced by Pseudomonas aeruginosa NCIM 5514. Bioresour Technol. 2016; 220:175-182.

37. Wang Q, Fang X, Bai B, Liang X, Shuler PJ, Goddard WAll, Tang Y. Engineering bacteria for production of rhamnolipid as an agent for enhanced oil recovery. Biotechnol Bioeng. 2007; 98:842-53.

38. Hosseininoosheri P, Lashgari HR, Sepehrnoori K. A novel method to model and characterize in-situ bio-surfactant production in microbial enhanced oil recovery. Fuel. 2016; 183: 501-511.

39. Sponza DT, Gök O. Effect of rhamnolipids on the aerobic removal of polyaromatic hydrocarbons (PAHs) and COD components from petrochemical wastewater. Bioresour Technol. 2010; 101(3):914- 
924.

40. Eraqi WA, Yassin A S, Ali A E, et al. Utilization of Crude Glycerol as a Substrate for the Production of Rhamnolipid by Pseudomonas aeruginosa. Biotechnology Research International, 2016; 3464509.

41. Zhao F, Jiang H, Sun $\mathrm{H}$, et al. Production of rhamnolipids with different proportions of monorhamnolipids using crude glycerol and a comparison of their application potential for oil recovery from oily sludge. RSC Adv, 2019; 9(6): 2885-2891.

42. Pobletecastro I, Wittmann C, Nikel PI. Biochemistry, genetics and biotechnology of glycerol utilization in Pseudomonas Microb Biotechnol, 2020; 13(1): 32-53.

43. Biebl H, Menzel K, Zeng AP, Deckwer WD. Microbial production of 1,3-propanediol. Appl Microbiol Biotechnol, 1999; 52: 289-297.

44. Hauser G, Karnovsky ML. Rhamnose and rhamnolipide biosynthesis by Pseudomonas aeruginosa. $\mathrm{j}$ Biol Chem, 1957; 224(1): 91-105.

\section{Figures}



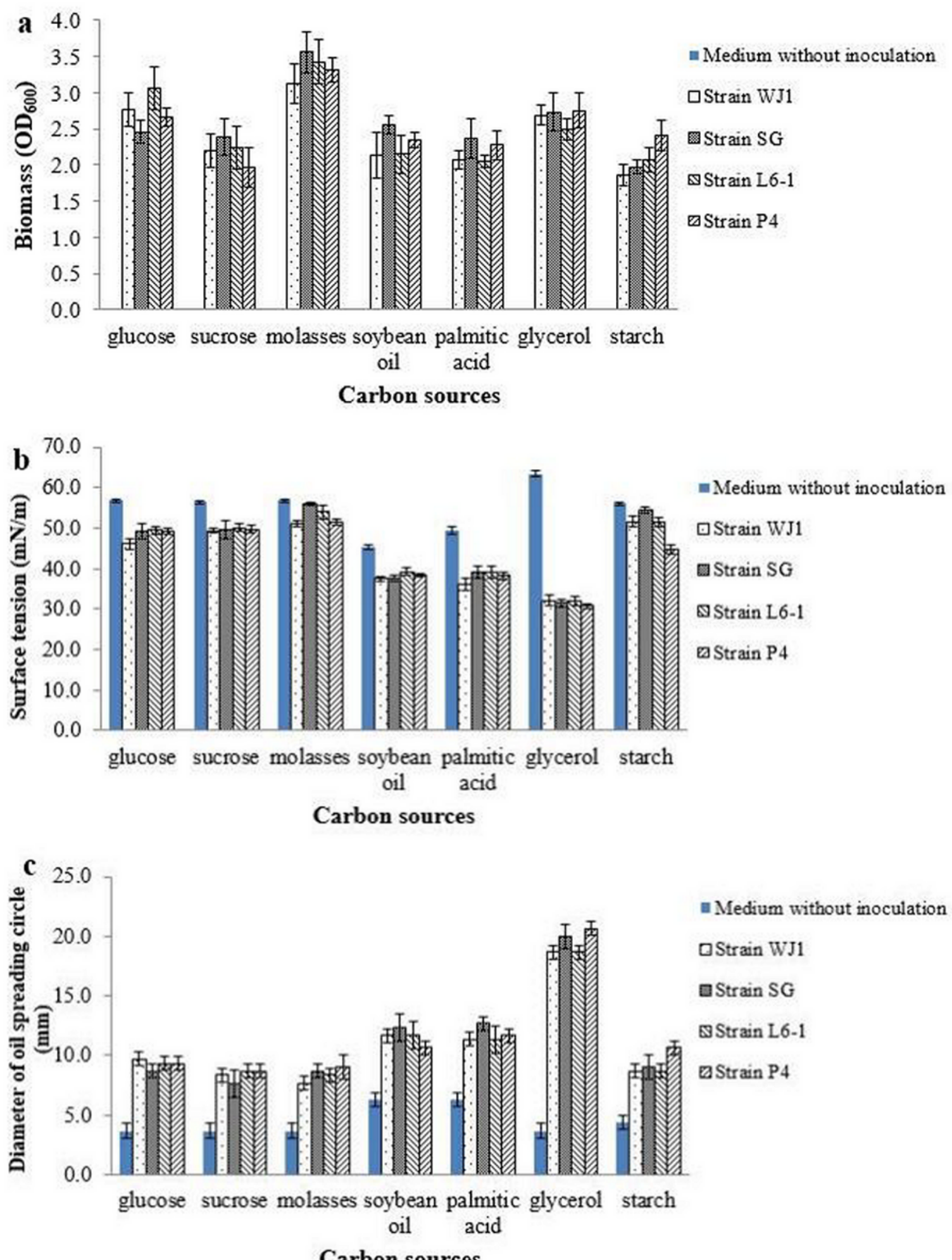

Figure 1

Anaerobic growth and production of rhamnolipids by four P. aeruginosa strains using different carbon sources: a) biomass (OD600), b) surface activity and c) oil spreading activity. Strain P. aeruginosa SG was used as positive control. 

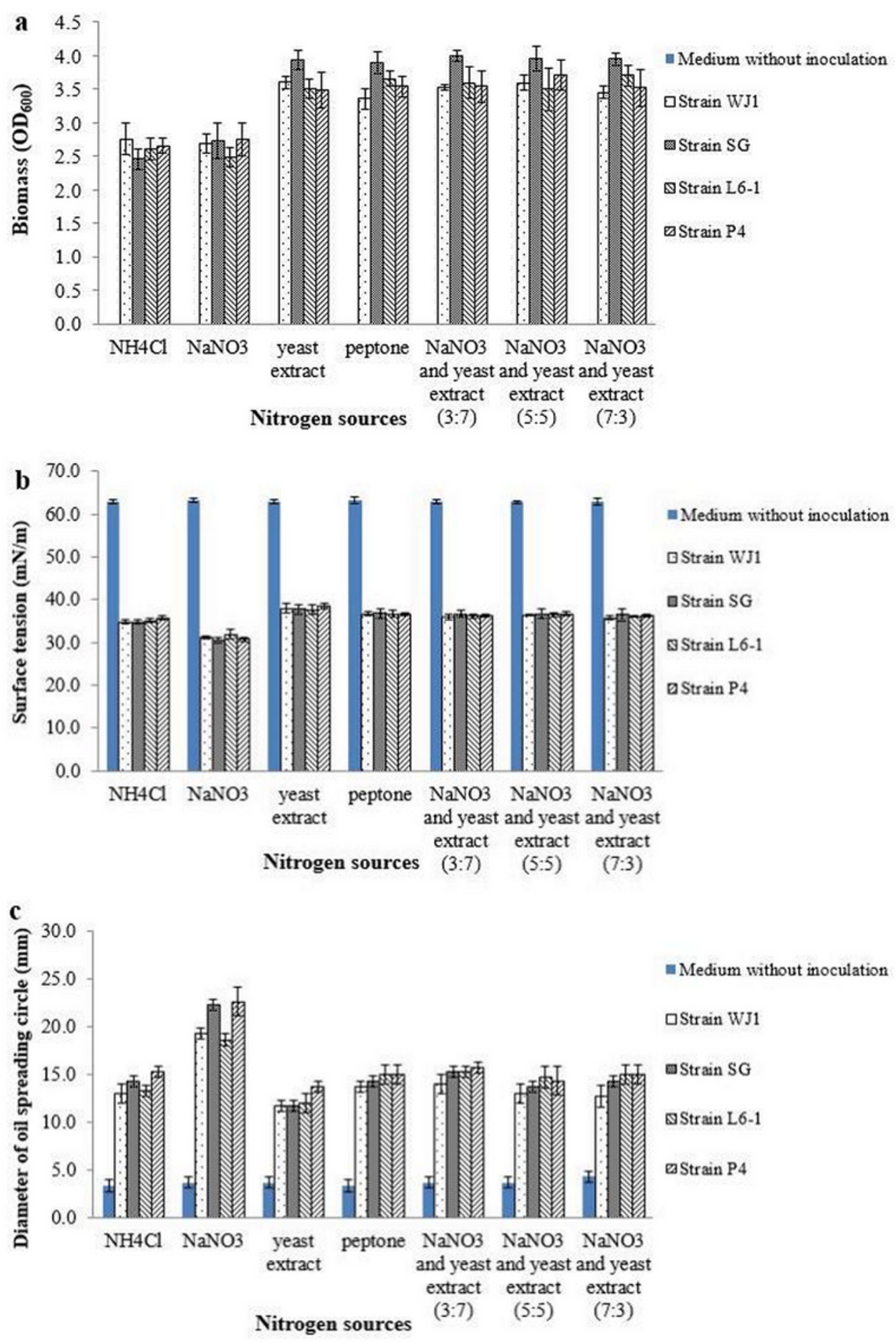

Figure 2

Anaerobic growth and production of rhamnolipids by four P. aeruginosa strains using different nitrogen sources: a) biomass (OD600), b) surface activity and c) oil spreading activity. Strain P. aeruginosa SG was used as positive control. 


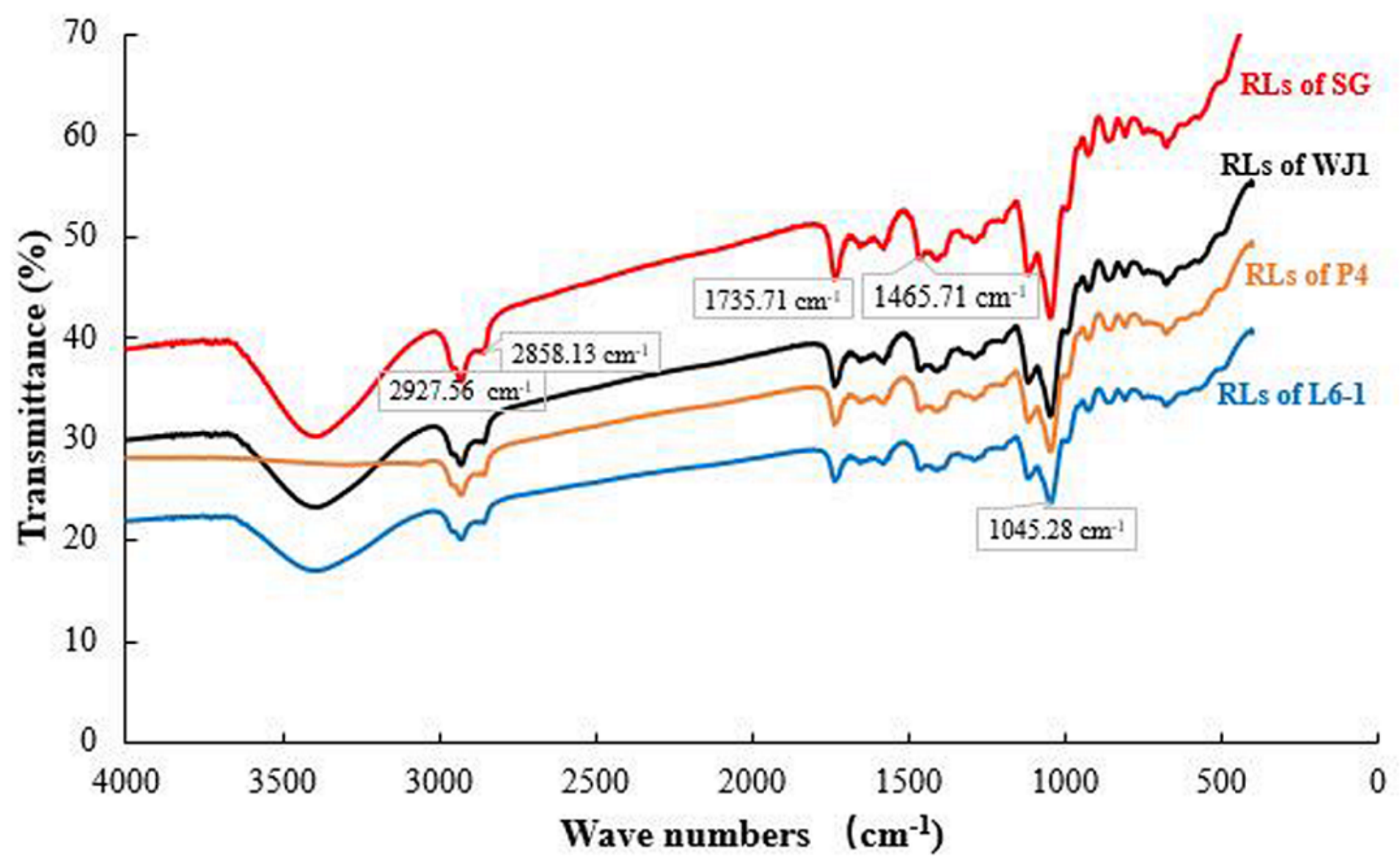

Figure 3

Fourier Transform infrared (FTIR) spectra analysis of anaerobically produced rhamnolipids from four P. aeruginosa strains using glycerol and nitrate. Anaerobically produced rhamnolipids by strain SG was used as control. 


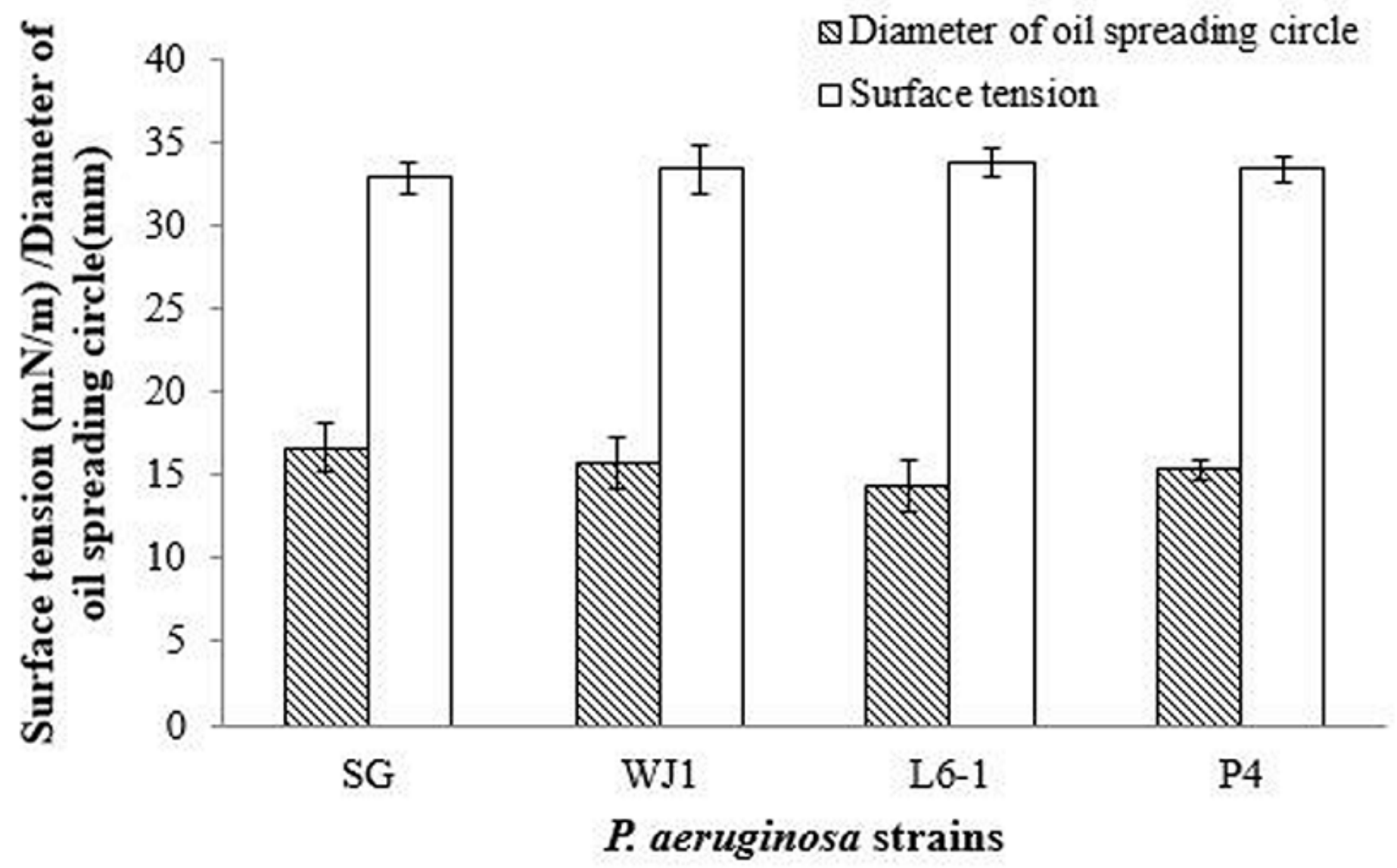

Figure 4

Anaerobic production of rhamnolipids by four P. aeruginosa strains using crude glycerol. 

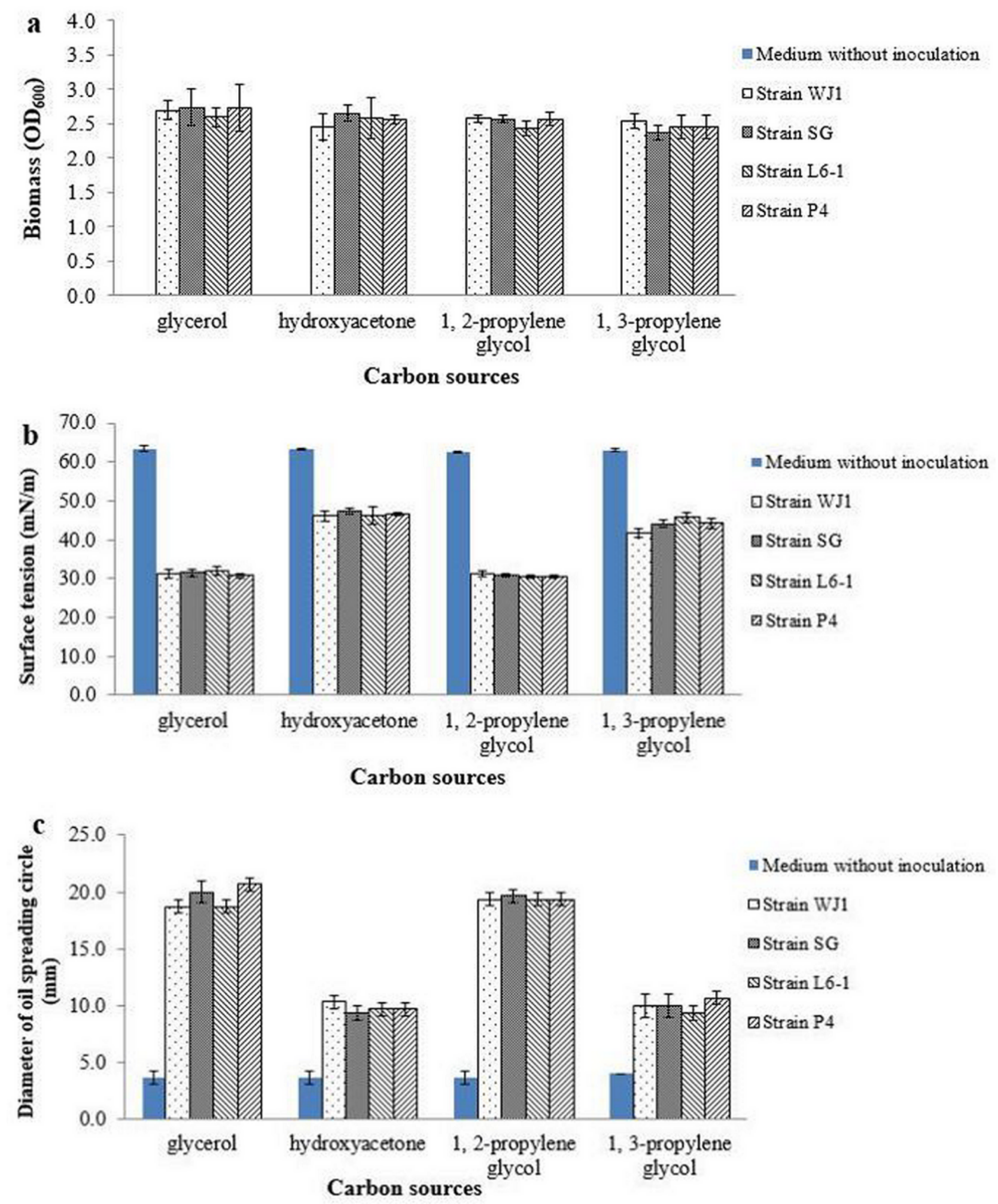

\section{Figure 5}

Anaerobic growth and production of rhamnolipids by four P. aeruginosa strains using the possible metabolic intermediates and analogues of glycerol as carbon sources: a) biomass (OD600), b) surface activity and c) oil spreading activity. Glycerol was used as positive control. 